\title{
Optimization of Superstructured Fiber Bragg Gratings for Microwave Photonic Filters Response
}

\author{
J. S. Leng, W. Zhang, and J. A. R. Williams
}

\begin{abstract}
The microwave photonic responses of superstructured fiber Bragg gratings in combination with dispersive fiber are investigated theoretically and experimentally. The superstructured gratings are optimized, taking account of the spectral response of the broad-band source, Erbium-doped fiber amplifier, and optical tunable filter to achieve a filter response with sidelobe suppression of more than $60 \mathrm{~dB}$.
\end{abstract}

Index Terms-Microwave photonic filter, superstructure fiber Bragg grating (SFBG).

\section{INTRODUCTION}

D EVICES OFFERING the capability to process microwave/millimeter wave signals in the optical domain have attracted much interest [1], [2]. Commonly, these processing devices use fiber delay lines to produce the filter taps. The tap lengths differ much by precisely set multiples of the unit delay and must be correctly weighted to get the desired response; more taps are required to increase the $Q$ factor and improve the rejection level and suppression of sidelobes. It becomes difficult to manage the adjacent tap lengths with sufficient accuracy as their number increases.

Fiber grating arrays have also been widely used to establish the taps [3]-[5]. These enable increased flexibility and programmability in the design of the microwave delay line filter, and it is easier to precisely position fiber Bragg gratings than match fiber lengths the task still becomes difficult as the number of taps required increased.

A solution to this problem is to use a superstructured fiber Bragg grating (SFBG) which creates a precise comb of weighted optical peaks which are all modulated by the same microwave signal. A dispersive fiber then delays each peak by a precise amount after which the peaks are recombined on a photodiode. This contrasts to other methods where the delay is introduced by lengths of fiber. With such devices, $45 \mathrm{~dB}$ of sidelobe suppression has been demonstrated [4] using a single fixed nonoptimum set of tap weights. In this work, the microwave response of an SFBG is investigated using different tap profiles, and using a Voigt function profiled SFBG a sidelobe suppression level larger than $60 \mathrm{~dB}$ is demonstrated. We believe this is the highest rejection level demonstrated for a passive optical delay line filter.

\section{PRINCIPLE}

Generally, in a microwave photonic system using direct modulation, the microwave filtering based on optical delay line is

Manuscript received December 3, 2003; revised February 27, 2004.

The authors are with the Photonics Research Group, Aston University, Birmingham B4 7ET, U.K. (e-mail: j.leng@Aston.ac.uk).

Digital Object Identifier 10.1109/LPT.2004.828363 realized by incoherently summing $N$ optical signals with an optical path increment $n L_{0}$ to produce a bandpass response at the microwave frequencies $f=i\left(c / n L_{0}\right), i=0,1,2 \ldots$ The microwave signals incoherently add at these frequencies, thus, a bandpass response can be achieved using $N$ optical fiber delay lines.

An SFBG is a Bragg grating with a periodically varying low spatial frequency modulation envelope superimposed upon it. Such modulation can easily and precisely be performed during fabrication of the devices. In the weak coupling coefficient limit, the grating reflectivity $\rho^{2}$ can be approximated as the Fourier transform

$$
\rho^{2}=\left|\int_{-L / 2}^{L / 2} \kappa(z) e^{j 2 \delta z} d z\right|^{2}
$$

where $\kappa(z)$ is the coupling coefficient distribution along the grating and $\delta$ the detuning. Every Fourier component of the modulation profile contributes a reflectivity peak to the reflection spectrum of the SFBG [6] and the wavelength spacing of the Bragg peaks is given by

$$
\Delta \lambda=\frac{\lambda^{2}}{2 n \Lambda}
$$

where $\lambda$ is the Bragg wavelength, and $\Lambda$ the period of the low frequency envelope. By combining such a device with a linearly dispersive medium, microwave photonics filtering can be achieved with a series of optical taps with a unit time delay determined by the product of the wavelength spacing $\Delta \lambda$ and the dispersion introduced by the medium. All the taps delays are, therefore, intrinsically set with precision. The relative strength of the taps is determined by the profile of each period of the sampled grating. If we make each section weak enough and apodise each section of the sampled grating, then the overall profile of the grating can be designed from (1).

\section{EXPERIMENTS AND RESULTS}

In these experiments, SFBGs with different periods, envelopes, and duty cycle were fabricated using the scanning phase mask technique [6] which enables accurate control of the grating coupling coefficient along its length and, thus, the resultant spectrum of the SFBG. In particular total length of the SFBG, duty cycle (the ratio of individual grating length to the sampling period), and period were optimized in these experiments. A natural apodization was realized for each section due to the modulation profile being convolved with the ultraviolet beam profile used in fabrication. In order to meet 


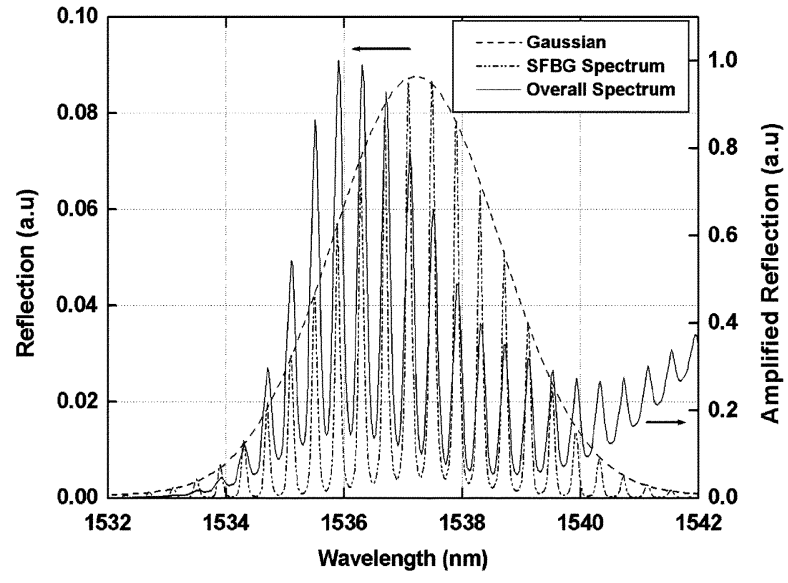

Fig. 1. Reflection spectrum of a Gaussian apodised SFBG.

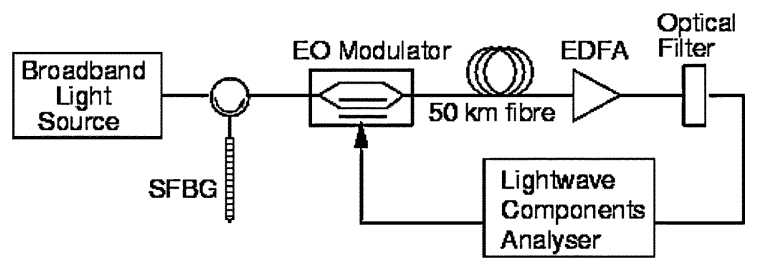

Fig. 2. Schematic illustration of experimental arrangement.

the requirement of (1), the grating has to be weak. Although a weak grating will reduce the available optical intensity, this can easily be compensated by using an Erbium-doped fiber amplifier (EDFA).

Fig. 1 shows the typical refiection spectrum obtained of an SFBG with a Gaussian envelope profile. The length of the grating was $48 \mathrm{~mm}$, with 24 samples created in hydrogen loaded single-mode fiber by modulating the laser with a duty cycle of $7.5 \%$, during fabrication. The SFBGs were then annealed at $90{ }^{\circ} \mathrm{C}$ for $48 \mathrm{~h}$ in an oven. This resulted in 22 peaks in the refiection spectrum (microwave taps) with a wavelength spacing of $0.4 \mathrm{~nm}$ between adjacent peaks.

The experimental arrangement illustrated in Fig. 2 was used to investigate the microwave responses of such SFBGs. A broad-band optical signal was refiected from the SFBG through a fiber circulator and then amplitude modulated at microwave frequencies using an electrooptic modulator. The modulated light was launched into $50 \mathrm{~km}$ of single-mode fiber used as a dispersive medium to provide the proper unit time delays. The light was then amplified to compensate for losses in the fiber and modulator using an EDFA and the microwave response measured for varying modulation frequencies using a lightwave components analyzer.

Fig. 3 shows the expected simulated microwave response for a filter with the optical response function shown in Fig. 1, illustrating that the sidelobes in the filter response are expected to be less than $-60 \mathrm{~dB}$ with respect to the main transmission peak. However, in the experiment, a contrast ration between main peak and sidelobes of only $-40 \mathrm{~dB}$.

In the experimental system, we have to account for the optical responses of the broad-band light source and the EDFA which results in a less ideal overall optical response. Therefore, optical filters can be used to compensate this nonideal response,

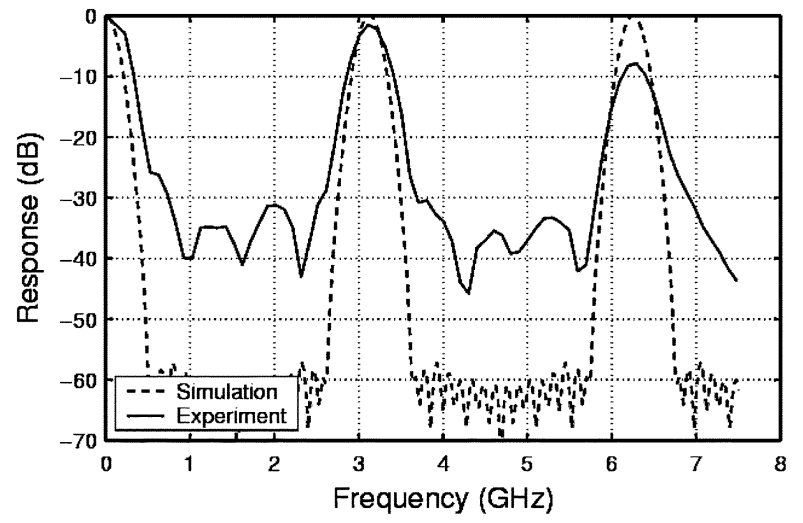

Fig. 3. Simulated microwave response corresponding to SFBG in Fig. 1.

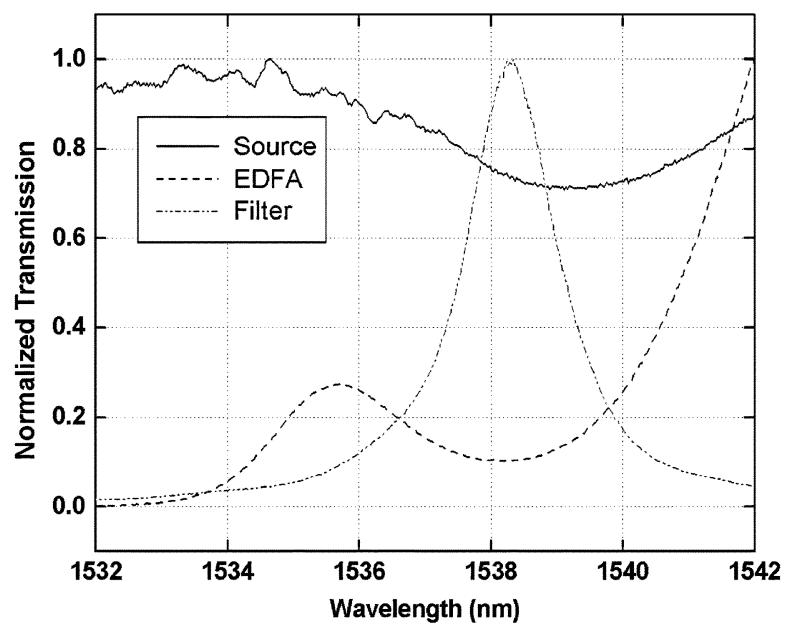

Fig. 4. Optical spectra of the broad-band source, EDFA, and a tunable filter.

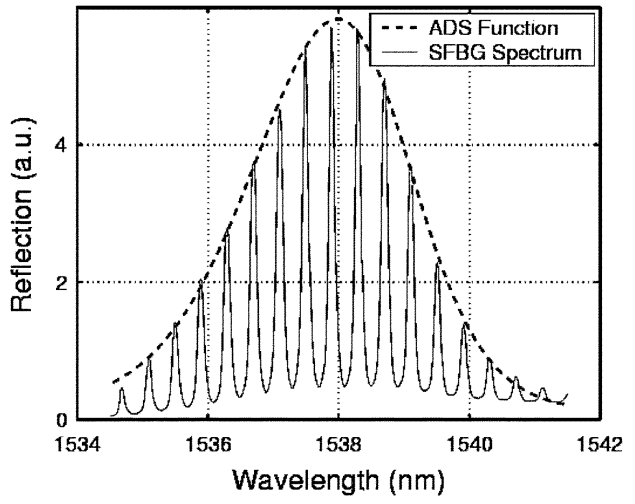

Fig. 5. Resultant spectrum of an SFBG with ADS function profile.

as shown in Fig. 2. The optical spectra of broad-band source, EDFA, and tunable filter are shown in Fig. 4. The use of tunable filters enables flexible control over the overall optical spectrum and, thus, the microwave frequency response. As an example in Fig. 5, we show an asymmetric double sigmoidal (ADS) reflection spectrum obtained by combining a 2.2-nm optical filter with a Gaussian profiled SFBG.

In Fig. 6, we show the microwave responses obtained using SFBGs with different profiles in combination with a tunable optical filter. The solid lines are the experimentally measured response and the dashed lines the numerical predictions. 


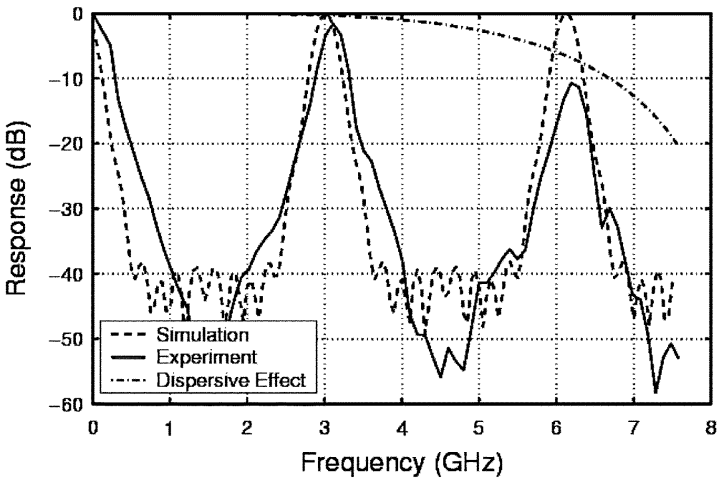

(a)

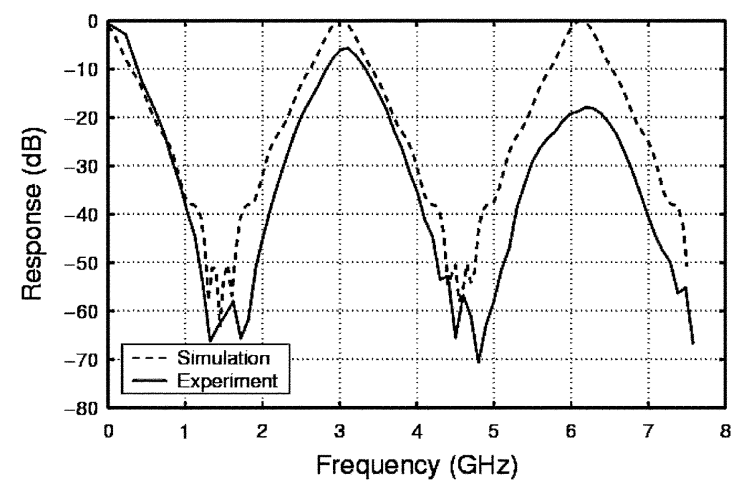

(b)

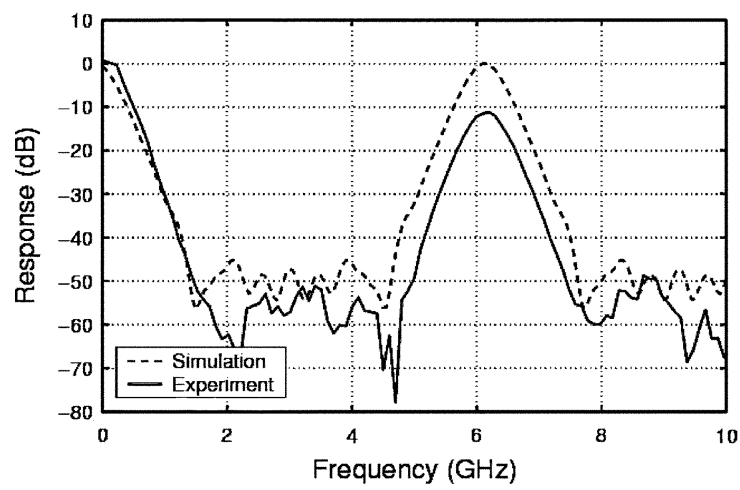

(c)

Fig. 6. SFBGs microwave responses with reflection profiles of (a) ADS function, (b) Voigt function, and (c) LogNormal function.

Fig. 6(a) shows the microwave response of using a sigmoidal optical spectral profile and 0.4-nm wavelength spacing. The free spectral range (FSR) of bandpass response is $3.1 \mathrm{GHz}$, corresponding to a unit time delay of $320 \mathrm{ps}$ at a wavelength of $1535 \mathrm{~nm}$. The experimental result shows a rejection level larger than $-50 \mathrm{~dB}$. It is noted that some of the measured passband levels clearly are lower than the simulation results. This degradation is attributed to the dispersion-induced fading [2] which causes a power penalty at specific frequencies and fiber lengths. The dashed-dotted line in Fig. 6(a) shows this dispersive response for the 50-km fiber used, from which we can see that the change in passband levels closely match the amplitude response of the fiber. The finite width of the optical peaks of the SFBG will result in an additional power penalty to frequency response. The larger peak width will produce larger power penalty.

In Fig. 6(b), we show the simulated and experimental results for a Voigt function optical spectral profile, which shows a rejection level of $-60 \mathrm{~dB}$, the highest rejection level for a passive optical delay line filter obtained to date as far as we are aware. However, the rolloff of the microwave passband profile is quite slow compared with the FSR in this case. By reducing the wavelength spacing between optical spectral peaks, thus reducing the time delay between the taps, we can increase the FSR. Fig. 6(c) is illustrative of this and shows a bandpass response with a 52-dB rejection level and a 6.2-GHz FSR from a grating with a lognormal function profile and $0.2-\mathrm{nm}$ wavelength spacing.

Increasing the number of the channels will increase the $Q$ factor and rejection level [3]. However, a larger number of taps will usually cover a larger wavelength range, such that the fiber dispersion slope becomes significant and results in mismatches in the delays between the tabs and, thus, a degraded filter response.

\section{CONCLUSION}

In this work, SFBGs have been used together with a broad-band source and a section of dispersive fiber to synthesise microwave photonic transversal filters. The unit delay between taps in the filter is controlled by product of the wavelength spacing between taps and the fiber dispersion. The tap weights have optimized by considering the response envelope of the SFBG together with that of the broad-band source, EDFA, and a tunable optical filter. A number of response functions were synthesised and we have demonstrated a $60-\mathrm{dB}$ rejection level, for a bandpass microwave filtering response, the highest to date for this type of filter.

\section{REFERENCES}

[1] D. Hunter and R. Minasian, "Microwave optical filters using in- fiber Bragg grating arrays," IEEE Microwave Guided Wave Lett., vol. 6, pp. 103-105, Feb. 1996.

[2] H. Schmuck, "Comparison of optical millimeter wave system concepts with regard to chromatic dispersion," Electron. Lett., vol. 31, no. 21, pp. 1848-1849, 1995.

[3] W. Zhang, G. Yu, and J. Williams, "Tap multiplexed optical transversal filter by using fiber gratings," Electron. Lett., vol. 36, no. 20, pp. 1708-1710, 2000.

[4] B. A. L. Gwandu, W. Zhang, and J. A. R. Williams, "Microwave photonic filtering using a profiled superstructured fiber Bragg grating and dispersive fiber," in Proc. Microwave Photonics 2000, Awaji, Japan, 2002, Paper T2-6.

[5] D. B. Hunter and R. Minasian, "Microwave optical filters using in-fiber Bragg grating arrays," IEEE Microwave Guided Wave Lett., vol. 6, pp. 103-105, Feb. 1996.

[6] B. Eggleton, P. Krug, L. Poladian, and F. Ouellette, "Long periodic superstructure Bragg gratings in optical fibers," Electron. Lett., vol. 30, no. 19, pp. 1620-1622, 1994. 